\title{
Wavelet-Based Power Network Disturbance Identifying Part II: Application in Large Scale Power System
}

\author{
Gang Chen ${ }^{1, \mathrm{a} *}$, Bo Zhou ${ }^{1, \mathrm{~b}}$, Hua Zhang ${ }^{1, \mathrm{c}}$, Lijie Ding ${ }^{1, \mathrm{~d}}$ \\ ${ }^{1} 24$ Qinghua Road, Qingyang District, Chengdu, Sichuan, 610072, CHINA \\ agangchen08@gmail.com, bzbv_s@126.com, 'czhanghua002@163.com, ding_lijie@163.com
}

Keywords: Wavelet multi-resolution analysis, wavelet coefficient, wide-area measurement system, disturbance.

\begin{abstract}
It is of great help to take proper anticipatory actions to eliminate the disturbances that identifying disturbances timely and accurate, avoiding blackouts. In this paper, the wavelet multi-resolution analysis based method is introduced to identify power system disturbances. The effectiveness of the method is addressed by applying to a real power system in Western China. After that, based on the method, a software system based on the proposed method is developed. In this paper, the design and implementation of the system is introduced. The system receives real-time frequency signals from wide area measurement system (WAMS), identifying disturbances online. The system has been running in actual power system, achieving engineering application. The measured disturbances further validate the effectiveness and feasibility of the method and software.
\end{abstract}

\section{Introduction}

With the interconnection of power grids, the scale of power network becomes to be more and more larger, as a result of which, a small and local disturbance might spread to far away from the disturbance source, even leading to blackouts. Therefore, it is of great meaning to identify the source and occur time of disturbance, that may help the operators to take actions to eliminate the disturbance, preventing of the occurrence of blackouts. With the phasor measurement Units (PMUs) based wide area measurement system (WAMS), the operational condition of the power grid can be monitored continuously and the dynamics of the power system can be recorded [1].

Wavelet transform (WT) is a powerful and systematic way of analyzing the abrupt-changing feature of signals, belonging to a type of time-frequency-domain analysis [1]. In [3], a wavelet-based power network disturbance identifying method has been proposed, and case study is carried out on an IEEE 39-bus power system. In this paper, the application of the method to a real large scale power system is described, and a software system based on the proposed method is also developed.

In this paper, the application in large scale power system of a wavelet-based power network disturbance identifying method is described. First, the wavelet multi-resolution analysis based method is reviewed in Section 1. Then, the application of the method in a power system in Western China, is described in Section 2. The design and implementation of a platform of power network disturbance identifying is introduced in Section 3. The conclusion is discussed in Section 4.

\section{Review of the Power Network Disturbance Identifying Based on MRA}

According to reference [3], the basic process of the disturbance identification method proposed in this paper can be concluded as follows:

Step 1: typical disturbances, including generation loss, load drop, short circuit fault, etc, are simulated. Frequency signals are recorded by PMUs, as the frequency signal is a very good signal to reveal the disturbance characteristic [4].

Step 2, a library of wavelet functions is established. Since there are too many wavelet functions, it is hard to enumerate all the existing wavelet functions. In this paper, the DB family of wavelet function are selected as candidates [5]. 
Step 3, for each disturbance, WT-based MRA is applied to the measured frequency signals to obtain wavelet coefficients (WCs) with each of the wavelet functions in the database established in Step 1, respectively. Using the following equation, the energy contained in WCs can be obtained:

$$
E_{i}=\sum_{j=1}^{n_{i}}\left|d_{i j}\right|^{2}
$$

where $i$ is the scale; $j$ is the number of points contained in each detail.

According to Eq. 1, the WC's energy of the disturbance can be obtained by summing up the energies of frequency signal measured by each PMU, since there are several frequency responses at the PMU locations. Therefore, the selection of wavelet function should obtain the maximum WC's energy for specific disturbances.

Step 4, having the function in Step 3, we can get the total energy of all decomposition levels by:

$$
E=\sum_{i=1}^{N} E_{i}
$$

where $N$ denotes the decouple scale, $i$ is the total energy of all decomposition levels. The decomposition scale can be selected by having the maximum energy according to Eq. 2.

Step 5, using the selected wavelet function in Step 3 and decomposition scale in Step 4, frequency signals measured by PMUs are identified by WT-based MRA, obtaining the highest WC for each PMU sub-station. Then, the time and location of the disturbance is estimated.

\section{Application in Large Scale Power System}

In this section, the method is applicated in a real power grid in western China [6], the geographical system connection diagram of which is shown in Fig. 1. PMUs are located at the buses which are dotted red in Fig. 1.

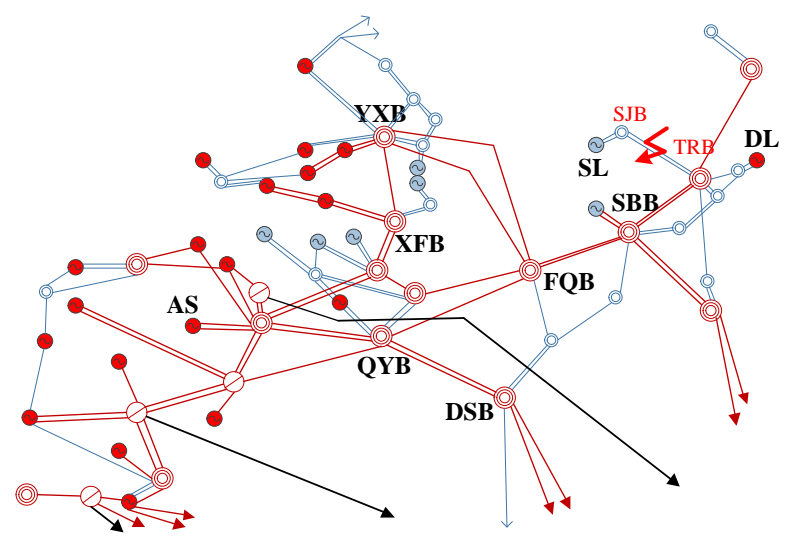

Fig. 1 The geographical system connection diagram for one power grid in Western China

In order to determine the appropriate wavelet function and its decomposition scale, generator loss and a certain percentage of load loss disturbances at different buses were simulated in the BPA software, and the frequency siganls are recorded by PMUs. The maximun energy of WCs were obtained by using the wavelet function of ' $\mathrm{db} 2^{\prime}$. And the energy was concetrated in the 3rd layer of decomposition, with the choice of ' $\mathrm{db} 2$ '. Therefore, the selection of wavelet function and its decomposition scale are 'db2' and 3.

The identification result from the frequenies while considering 300MW generator loss at AS Bus are shown in Fig. 2.

It can be seen from Fig. 2, that, the appearence time of the maximum WC is just the time of the occurance of disturbance, when the generator loss disturbance occurs at Bus AS. The size of WCs from the signal recorded by different PMUs can be arranged as follows: AS, XFB, QYB, DSB, FQB, SBB, DL, SL, in which AS has the largest one. As a result, AS Bus can be considered as disturbance source. Compared Fig. 1, it can be found that, the arragement of WCs is coincided with the distance from the measure Bus the disturbance source of AS. It should be noted that there is just a PMU 
located at the disturbance soure. If not, the disturbance soure would be identified to XFB, which is the nearest Bus from AS. In other words, the distribution of PMU directly affects the accuracy of the disturbance source identification.

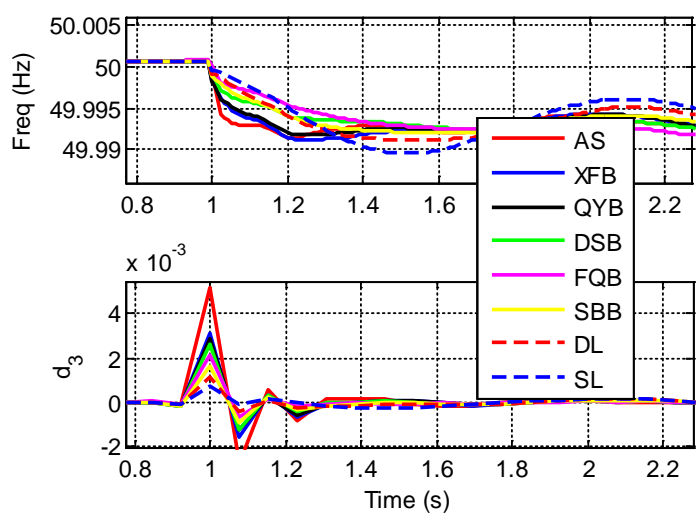

Fig. 2 Identification results for frequency responses to generation loss at AS\#1

The identification result from the frequenies while considering load loss at YX Bus are shown in Fig. 3. It can be seen from Fig. 3 that the identification results from frequency signals can reveal the disturbance source, which is the similar with the generator loss disturbance. If there is a PMU located at the disturbance source, the disturbance can be identified accurately, if not, the result is the Bus nearest to the disturbance source. In fact, in real power system, one cannot know where the disturbance occurs, therefore, the disturbacne source can be narrowed by using the method proposed in this paper.

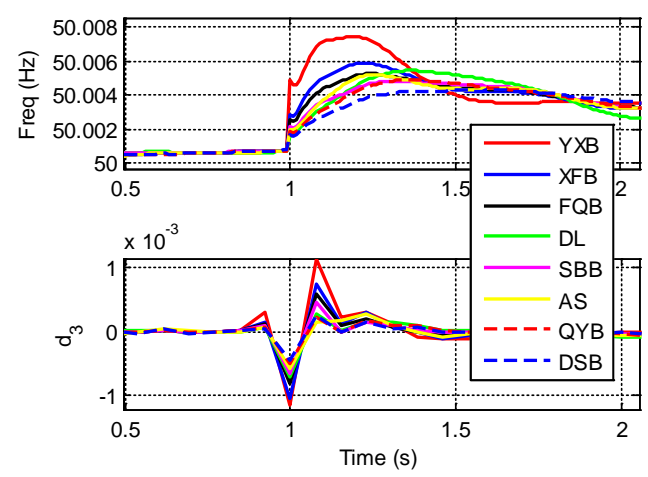

Fig. 3 Identification results for frequency responses to 300MW load loss at YXB

\section{Software Implementation in Real Power System}

In order to apply the proposed method to the real power system, a power network disturbance identification system (PNDIS) was developed, including the identification service module and disturbance source visualization workstations module. The structure of the PNDIS is shown in Fig. 4. In Fig. 4, the online identification service module identifies frequency sigals, which are obtained from the PMU master-station, by WT-based MRA. After that, the indentification results are transmitted to the visualization workstations, where the results are shown on the main interface which is shown in Fig. 5.

A generator loss disturbance at SL was detected in GZPG on Jul 22, 2010 and recorded by the WAMS. The data is used to issue the functions of visualization module and the benefits of the platform.

In Fig. 5, a map of the power system is used as the background of the left side of main interface. A PMU sub-station is doted as a cylinder with color on the map at just its location place. The darker red the color of the cylinder is, the closer the corresponding PMU to the disturbance source is. Also, the higher the cylinder is the closer the corresponding PMU to the disturbance source is. In other words, the darkest and highest cylinder of PMU is the closest site to the disturbance. In Fig. 8, which replayed the 200MW generator loss disturbance at SL on Jul 22, 2010, the highest and darkest cylinder of PMU at SL indicates that the disturbance is at SL. 


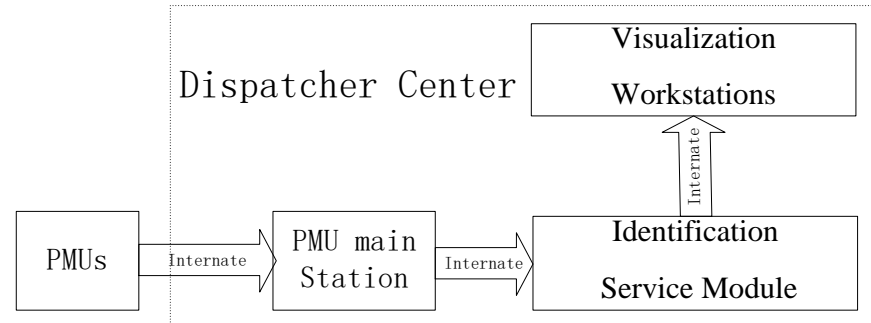

Fig. 4 Structure of the power network disturbance online identification system

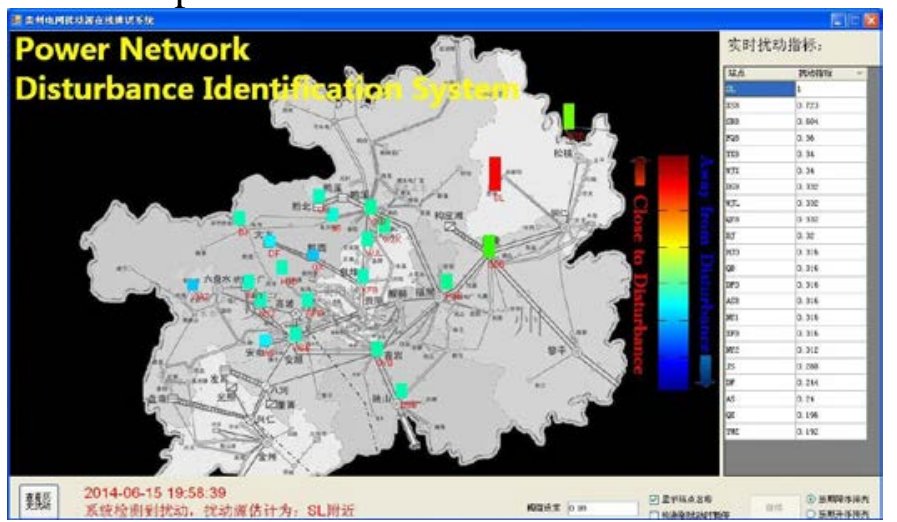

Fig. 5 Interface of the power network disturbance online identification system

\section{Conclusions}

In this paper, the wavelet-based power network disturbance identifying method is applied to a larege scale power system in Western China. The case studies get a further verification of the method. To apply the method in real power system, a software was developed and applied in Guizhou Power Grid in China. The running to the software confims the effectiveness of the method and the pratical of the software.

\section{References}

[1] REE J. D. L., CENTENO V., THORP J. S., et al. Synchronized phasor measurement applications in power systems. IEEE Trans. Smart Grid, 2010, 1(1): 20-27.

[2] MEI K. J., ROVNYAK S. M., and ONG C. M. Clustering-based dynamic event location using wide-area phasor measurements. IEEE Transactions on Power Systems, 2008, 23(2): 673-679.

[3] CHEN Gang, TANG Ming, DING Lijie, et al. Wavelet-Based Power Network Disturbance Identifying Part I: Theory and Method, 2015 6th International Conference on Advances in Circuits, Electronics and Micro-Electronics(CENICS 2015), Xi’an, China.

[4] ZHANG Y. C., MARKHAM P., XIA T., et al. Wide-Area Frequency Monitoring Network (FNET) Architecture and Applications [J]. IEEE Trans. Smart Grid, 2010, 1(2): 159-167.

[5] I. Daubechies: IEEE Trans. Inf. Theory Vol. 36, No. 5 (1990), p. 961-1006.

[6] CHEN Gang, SUN Yuan-zhang, CHENG Lin, et al. A novel PSS-online re-tuning method. Electric Power Systems Research. 2012, 91: 87-94. 